\section{Thoracic dog bite wounds in cats: a retrospective study of 22 cases}

\section{Anna Frykfors von Hekkel, Zoë Halfacree}

Royal Veterinary College, South Mimms, Hertfordshire, UK

\section{OBJECTIVES}

To compare clinical findings, investigate indications for thoracotomy and identify risk factors for mortality in cats with thoracic dog bite wounds.

\section{METHODS}

Medical records of cats with thoracic dog bite wounds presenting to a single institution between 2005-2015 were retrospectively reviewed. Data relating to clinical presentation, wound depth and management, radiographic findings and mortality were collected. Wound management was defined as conservative, exploratory or requiring thoracotomy. Statistical analyses were performed using Fisher's Exact, Mann Whitney and independent-samples t-test.

\section{RESULTS}

22 cats were included (55\% first opinion, $45 \%$ referral), of which eight cats $(40 \%)$ suffered penetrating wounds, two (10\%) deep wounds, five (25\%) full thickness skin wounds and five (25\%) partial thickness skin wounds. 16 cats $(73 \%)$ also suffered wounds elsewhere, most commonly to the abdomen. Neither an abdominal wound nor abdominal surgery was associated with mortality. Pneumothorax was the most common radiographic finding and was associated with presence of pseudo-flail chest $(p=0.050)$. Presence of pseudo-flail chest $(p=0.005)$, sternal fracture $(p=0.013)$ or penetrating wound $(p=0.001)$ was associated with decision to perform thoracotomy. There was no association between number of radiographic lesions and thoracotomy or mortality. The decision to perform thoracotomy was not associated with mortality. The overall mortality rate was $32 \%$. Cats that survived were significantly younger $(p=0.008)$ and lighter $(p=0.023)$ than cats that did not, with a median age and bodyweight of 30 months and $4.0 \mathrm{~kg}$, versus 102 months and $5.5 \mathrm{~kg}$, respectively.

\section{STATEMENT}

Clinical application of the presented data may facilitate both specialist and general practitioners' decision making in the management of feline thoracic bite wounds.
Congenital pleuroperitoneal hernia presenting as gastrothorax in five Cavalier King Charles Spaniel dogs and one British short hair cat

\section{Matteo Rossanese ${ }^{1}$, Mauro Pivetta ${ }^{2}$, Nuno Pereira Pereira ${ }^{3}$, Rachel Burrow ${ }^{1}$}

1 Small Animal Teaching Hospital, University of Liverpool, Neston, UK

2 North Downs Specialist Referrals, Bletchingley, UK

3 Klinik für Kleintiermedizin, University of Zurich, Zürich, Switzerland

\section{OBJECTIVES}

To describe the presentation, diagnostic findings, surgical treatment and outcome of pleuroperitoneal diaphragmatic hernia (PIPDH) and gastrothorax in five dogs and one cat.

\section{METHODS}

Five Cavalier King Charles Spaniel (CKCS) dogs and one British Short Hair cat ranging between 2.5 and 18 months of age were examined for acute onset of respiratory distress with no history of previous trauma.

\section{RESULTS}

Thoracic radiographs demonstrated a diaphragmatic hernia in all the cases. Tension gastrothorax was present in all the CKCS dogs, visible as a distended stomach occupying the left caudal thoracic cavity. Exploratory midline coeliotomy confirmed a congenital PIPDH with herniation and dilation of the stomach. The hernia configuration was remarkably consistent in all the cases, with a defect affecting the left diaphragmatic crus.

\section{STATEMENT}

Congenital PIPDH is a rare condition caused by a defect in the dorsolateral diaphragm. Defects of the left crus of the diaphragm could result in herniation of the stomach into the thoracic cavity with possible subsequent tension gastrothorax. Cavalier King Charles Spaniels may have a genetic predisposition to this condition. Tension gastrothorax is an acute life-threatening consequence of gastric herniation through a diaphragmatic defect that must be promptly recognised and surgically treated. Initial management involves decompression of the stomach with subsequent surgery to reduce the stomach, and to repair the diaphragmatic defect. As this case series reported, prompt surgical management can lead to a successful outcome. 